\title{
CLIFFORD SEMIGROUPS AND MONOTONICITY
}

\author{
T.E. HAYS
}

\begin{abstract}
A semigroup $S$ is said to be monotone if its binary operation is a monotone function from $S \times S$ into $S$. This paper utilizes some of the known algebraic structure of Clifford semigroups, semigroups which are unions of groups, to study topological Clifford semigroups which are monotone. It is shown that such semigroups are preserved under products, homomorphisms, and, under certain conditions, closures. Necessary and sufficient conditions for monotonicity of groups, paragroups, bands, compact orthodox Clifford semigroups, and compact bands of groups are developed.
\end{abstract}

\section{Introduction}

Considerable work has been done in recent years on the structure of semigroups which are the unions of groups. The portion of this work that is of interest to this paper is that of Clifford and Preston [2], Petrich [7], and Leech [6]. We will utilize these algebraic results to study topological semigroups which are the unions of groups, known as Clifford semigroups, and on which the binary operation is a monotone function. We show that such semigroups are preserved under products, homomorphisms, and compact closures. We also develop necessary and sufficient conditions for certain clifford semigroups to be monotone. We show, for example, that compact orthodox Clifford semigroups are monotone if and only if the

Received 18 December 1984.

Copyright Clearance Centre, Inc. Serial-fee code: 0004-9727/85 $\$ \mathrm{~A} 2.00+0.00$. 
maximal subgroups are connected and the subsemigroup of idempotents is monotone.

\section{Notation and terminology}

Throughout this paper the term "semigroup" unless otherwise specified, will mean topological semigroup. Our standard references will be $\mathrm{Clifford}$ and Preston [2], Petrich [7], and Hocking and Young [4]. Definitions of undefined terms may be found in these references.

DEFINITION 2.1. A function $f: X \rightarrow Y$, where $X$ and $Y$ are spaces, is monotone if $f^{-1}(y)$ is a connected subset of $X$ for each $y$ in $Y$.

DEFINITION 2.2. A semigroup $S$ is monotone if the binary operation on $S$ is a monotone function from $S \times S$ into $S$.

The standard Green's equivalence relations, $H$ and $J$, will be defined as in Petrich [7]. The corresponding equivalence classes will be denoted by $H(s)$ and $J(s)$, where $s$ is an element of the semigroup.

It is well known that $J$ is an algebraic congruence on a Clifford semigroup and that each J-class is algebraically a completely simple semigroup or equivalently, a paragroup (see Clifford and Preston [2], Theorem 4.6 and p. 46).

\section{Preservation of monotonicity}

In this section we focus our attention on the preservation of monotonicity of Clifford semigroups under products, homomorphisms, and compact closures.

THEOREM 3.1. Let $S_{i}$ be a semigroup for each $i$ in the set $I$. Then the product semigroup $S=X S_{i}$ is monotone if and only if $S_{i}$ is monotone for each $i$ in $I$.

Proof. Let $m_{i}$ denote the binary operation of $S_{i}$ for each $i$ in $I$ and $m$ denote the operation on $S$. Letting $\left(s_{i}\right)$ denote an element of $S$ and, defining $f$, 


$$
f: m^{-1}\left(\left(s_{i}\right)\right) \rightarrow X m_{i}^{-1}\left(s_{i}\right)
$$

by

$$
f\left(\left(t_{i}\right),\left(u_{i}\right)\right)=\left(\left(t_{i}, u_{i}\right)\right)
$$

It is clear that the range of $f$ is appropriate and that $f$ is a homeomorphism onto. Thus it follows that the domain of $f$ is connected if and only if $m_{i}^{-1}\left(s_{i}\right)$ is connected for each $i$ in $I$, that is, $S$ is nonotone if and only if each $S_{i}$ is monotone.

THEOREM 3.2. Let $f: C \rightarrow S$ be a homomorphism of the monotone clifford semigroup $C$ onto the semigroup $S$. Then $S$ is a monotone clifford semigroup.

Proof. That $S$ is algebraically a Clifford semigroup is well known and easily checked. Let $y$ be an element of $S$ with $y$ in $H(e)$, $e^{2}=e$ and let $B=\{x$ in $C: f(x)=y\}$. Consider the following set $U(f \times f)\left[m_{C}^{-1}(x)\right]$. It is straightforward to show that this set is precisely $m_{S}^{-1}(y)$. Note that if $f(x)=y$ then $f(g)=e$ where $x$ is in $H(g)$ with $g^{2}=g$. Thus $(y, e)$ is in $(f \times f)\left[m_{C}^{-1}(x)\right]$ for each $x$ in $B$ and thus $m_{S}^{-1}(y)$ is connected, that is, $S$ is monotone.

THEOREM 3.3. If $C$ is a monotone Clifford subsemigroup of the compact semigroup $T$, then $C^{*}$ is monotone, where * denotes closure.

Proof. Showing that $C^{*}$ is a Clifford semigroup is again a straightforward algebraic and topological argument. Let $y$ be in $C^{*}$ with $y$ in $H(e), e^{2}=e$ and suppose $s t=y$ with $s$ in $H(u), t$ in $H(v)$, and $u^{2}=u, v^{2}=v$. Since $s$ and $t$ are in $C^{*}$ we can assume without loss of generality that there are nets $\left(s_{i}\right)$ and $\left(t_{i}\right)$ in $c$ which converge to $s$ and $t$ respectively. The function $E(x)=f$ if and only if $x$ is in $H(f)$ is continuous (see Hoffmann and Mostert, [5], p. 44). Thus the net $E\left(s_{i} t_{i}\right)$ converges to $e$. Considering the set lim sup $m_{C}^{-1}\left(s_{i} t_{i}\right)$, we see that this set is connected (see Hocking and 
Young [4], p. IOI) and contains $(s, t)$ and $(e, y)$ since for each $i$, $\left(s_{i}, t_{i}\right)$ and $\left(E\left(s_{i} t_{i}\right), s_{i} t_{i}\right)$ are in $m_{C}^{-1}\left(s_{i} t_{i}\right)$. The result will be established if we show that this lim sup is a subset of $m_{C^{*}}^{-1}(y)$.

Suppose $(w, x)$ is in lim $\sup m_{C}^{-1}\left(s_{i} t_{i}\right)$ with $w x \neq y$. Since the binary operation on $C^{*}$ is continuous, there exist open sets $W, X$, and $V$ with $w$ in $W, x$ in $X$, and $y$ in $V$, respectively, such that $W X \cap V=\varnothing$. Since $\left(s_{i} t_{i}\right)$ converges to $y$, it is eventually in $V$. However, $W \times X$ is open in $C^{*} \times C^{*}$ containing $(w, x)$ and thus meets $\pi_{C}^{-1}\left(s_{i} t_{i}\right)$ for confinally many $i$, contrary to $W X \cap V=\varnothing$. Thus we must have $w x=y$ and $(w, x)$ in $m_{C^{*}}^{-1}(y)$.

REMARK. Theorems 3.2 and 3.3 are known to be true for other categories of monotone semigroups (see Hays, [3]) and it is a conjecture of the author that they are true for arbitrary monotone semigroups.

\section{Monotone Clifford semigroups}

In this section we investigate conditions that are imposed on a Clifford semigroup by the operation being monotone and also consider sufficient conditions for monotonicity.

THEOREM 4.1. Let $C$ be a monotone Clifford semigroup. Then

(i) $C$ is connected,

(ii) $J(e)$ is a monotone completely simple semigroup for each $e$ in $E(C)$, and

(iii) $H(e)$ is connected for each $e$ in $E(C)$.

Proof. (i). Let $x$ and $y$ be elements of $C$ and consider $m^{-1}(x y x y)$. By the monotonicity of the operation the set is connected and thus so is the set $P_{1} m^{-1}(x y x y)$ join $P_{2} m^{-1}(x y x y), P_{1}$ and $P_{2}$ are the projection maps from $C \times C$ into $C$. Noting that $x$ is in the former set and that $y$ is in the latter set, we have established that $C$ is connected. 
(ii). Let $e$ be in $E(C)$. Then by the known structure of $C$, $J(e)$ is a completely simple semigroup. To establish that it is monotone, let $x$ and $y$ be elements of $J(e)$ with $x$ in $H(u), y$ in $H(v)$, $x y$ in $H(z)$, and $u, v$, and $z$ in $E(C)$. Then by the monotonicity of $c, m_{C}^{-1}(x y)$ is a connected subset of $C \times C$ and thus the set

$$
\left\{(u s, t v):(s, t) \text { is in } m_{C}^{-1}(x y)\right\}
$$

is a connected subset contained in $J(e) \times J(e)$ containing $(x, y)$, and $(x y, z)$. Noting that $u s t v=x y$ for each $(s, t)$ in $m_{C}^{-1}(x y)$, we have that $J(e)$ is monotone.

(iii). From (ii) and (i), $J(e)$ is connected and since $H(e)=e J(e) e$, it follows that $H(e)$ is connected.

EXAMPLE. Letting $I$ be the unit interval under min-multiplication, $S=I \times\{0,1\}, M$ be the ideal $\{(0,0),(0,1)\}$. Then $S / M$ is a connected Clifford semigroup with $H(e)=J(e)$ connected for each idempotent in $S / M$, but $S / M$ is easily seen not to be monotone.

COROLLARY 4.2. A group is monotone if and only if it is connected.

COROLLARY 4.3. Every compact group can be imbedded in a compact monotone group.

In the material that follows a paragroup, denoted by $[X, G, Y]_{\sigma}$, will be defined as in Hoffmann and Mostert [5]. This result will be used in the establishment of Theorem 4.10.

THEOREM 4.4. A paragroup $[X, G, Y]_{\sigma}$ is monotone if and only if it is connected.

Proof. The necessity is clear by Theorem 4.1. To show the sufficiency, let $(x, g, y)$ be an element of the paragroup. Letting $m$ denote the binary operation, it is straightforward to demonstrate that $m^{-1}(x, g, y)$ is the set

$$
\left\{\left((x, s, y),\left(u,(v, u)^{-1} x^{-1} g, y\right)\right):(v, u) \text { is in } Y \times X \text { and } s \text { is in } G\right\} \text {. }
$$

Defining the function $F$ from $X \times G \times Y$ into $m^{-1}(x, g, y)$ by $F((u, s, v))=\left((x, s, v),\left(u,(v, u)^{-1} s^{-1} g, y\right)\right)$ it is easily seen that $F$ 
is continuous and onto. The connectedness of $X \times G \times Y$ and continuity of $F$ imply that $m^{-1}(x, g, y)$ is connected and thus establishing the result. COROLLARY 4.5. The minimal ideal of a compact connected semigroup is monotone.

Proof. It is well known and straightforward to verify that the minimal ideal of a compact connected semigroup is connected. It then follows from Hoffmann and Mostert ([5], p. 16) that this minimal ideal is also a paragroup. The result then follows from Theorem 4.4 .

DEFINITION 4.6. A band $B$ is a semigroup with each element idempotent. A band will be called left-upper connected if $\{y: x y=x\}$ is connected for each $x$ in the band. Similarly, a band will be called right-upper connected if $\{y: y x=x\}$ is connected for each element $x$ in the band. A semilattice, an abelian band, will be called upperconnected if $\{y: x y=x\}$ is connected for each element $x$ in it.

THEOREM 4.7. A band $B$ is monotone if and only if it is left and right upper-connected.

Proof. Suppose $B$ is monotone. Then for $x$ in $B, m^{-1}(x)$ is connected and thus so are the sets $P_{1} m^{-1}(x)$ and $P_{2} m^{-1}(x)$. Noting that $P_{1} m^{-1}(x)$ is precisely the set $\{y: y x=x\}$ and that $P_{2} m^{-1}(x)$ is likewise the set $\{y: x y=x\}$, the necessity is established. Now suppose that $B$ is left and right upper-connected. Then $u v=x$ implies that $u$ is an element of $\{y: y x=x\}$ and $v$ is an element of $\{y: x y=x\}$. These sets being connected imply that $\{(z u, v y): z x=x$ and $x y=x\}$ is a connected subset of $m^{-1}(x)$ containing $(u, v)$ and $(x u, v x)$. Furtherroore, the set $\{(x z, y x): z x=x$ and $x y=x\}$ is connected and contains $(x u, v x)$ and $(x, x)$. Noting that

$$
x z y x=(x y) z y(z x)=x(y z)(y z) x=x y z x=x \text {, }
$$

this latter set is also contained in $m^{-1}(x)$. Thus $(u, v)$ and $(x, x)$ lie in a connected subset of $m^{-1}(x)$. Since $(u, v)$ was arbitrary in $m^{-1}(x), m^{-1}(x)$ is connected and the band is monotone.

EXAMPLE. Let $B$ be a non-trivial set endowed with left trivial 
multiplication and the discrete topology. Then $B$ is right-upper connected but not left-upper connected and is, of course, not monotone.

The following result is immediate from the previous theorem and the definition.

COROLLARY 4.8. A semilatice is monotone if and only if it is upper connected.

We now turn our attention to bands of groups and orthodox clifford semigroups. It is in this area that most of the algebraic progress seems to have been made (see Clifford [1]).

DEFINITION 4.9. A Clifford semigroup $C$ is orthodox if its set of idempotents, $E(C)$, is a subsemigroup.

THEOREM 4.10. Let $C$ be an orthodox Clifford semigroup. If $E(C)$ is monotone and $H(e)$ is connected for each $e$ in $E(C)$, then $C$ is monotone.

Proof. Let $x$ be in $H(u), y$ in $H(v)$, and $x y$ in $H(z)$ with $u, v, z$ in $E(C)$. Then $E(C)$ monotone implies by Theorem 4.7 that the sets $R=\{w: w z=z\}$ and $L=\{t: z t=z\}$ are connected. Thus the set $B=\{(w x, y t): w$ in $R$ and $t$ in $L\}$ is connected with $(x, y)$ and $(z x, y z)$ in $B$ and $w x y t=w z(x y) z t=z(x y) z=x y$. Since $E(C)$ is monotone, it can be seen that $E(J(z))$ is connected by observing that if $e$ is in $E(J(z))$, then the following set $D$ given by

$$
D=e\left(P_{1} m_{E(C)}^{-1}(e z)\right) \cup\left(P_{2} m_{E(C)}^{-1}(e z)\right) z
$$

is a connected subset of $E(J(z))$ containing $e$ and $z$. Letting $X=E(J(z)) z$ and $Y=z E(J(z))$, then it is known that $J(z)$ is algebraically isomorphic to the paragroup $[X, H(z), Y]_{\sigma}$, where $\sigma(s, t)=s t$, under the mapping $f$ defined by $f(s, g, t)=s g t$ (see $\mathrm{Clifford}$ and Preston, [2], p. 94). The mapping is easily seen to be continuous, thus, by Theorems 4.4 and $3.2, J(z)$ is monotone. Hence $B \cup m_{J(z)}^{-1}(x y)$ is a connected subset of $m_{C}^{-1}(x y)$ containing $(x, y)$ and $(x y, z)$. Hence $C$ is monotone.

The following example indicates that while the above conditions are sufficient, they are not necessary. 
EXAMPLE. Let $R^{+}$be the non-negative reals under multiplication. Then $R^{+}$is a locally compact monotone Clifford semigroup with connected maximal groups, but $E\left(R^{+}\right)$is discrete and hence not monotone.

In the compact case, however, we have the following result.

THEOREM 4.11. Let $C$ be a compact orthodox Clifford semigroup. Then $C$ is monotone if and only if $E(C)$ is monotone and $H(e)$ is connected for each $e$ in $E(C)$.

Proof. The sufficiency follows from the previous theorem. The necessity of $H(e)$ being connected for each $e$ in $E(C)$ is just Theorem 4.1. To show that $E(C)$ is monotone, we show that the sets $R$ and $L$ defined as $R=\{y: y x=x\}$ and $L=\{y: x y=x\}$ are connected for each $x$ in $E(C)$, and then apply Theorem 4.7 to obtain the result. Since $C$ is assumed to be monotone, $m^{-1}(x)$ is connected and thus $P_{1} m^{-1}(x)$ is a connected subset of $C$. Since the mapping $E: C \rightarrow E(C)$ defined by $\vec{r}(s)=f$ if and only if $s$ is in $H(f)$ with $f^{2}=f$, is continuous (see Hofmann and Mostert, [5], p. 44), $F\left(P_{1} m^{-1}(x)\right)$ is a connected subset of $E(C)$ which is easily seen to be precisely the set $R$. Similarly $F\left(P_{2} m^{-1}(x)\right)$ is $L$ and connected. Thus $E(C)$ is monotone.

EXAMPLE. Let $I$ be the unit interval under min-multiplication, $S=I \times\{-1,1\}$, and $M$ be the ideal $\{(0,-1),(0,1)\}$. Then $S / M$ is a compact orthodox Clifford semigroup with $E(S / M)$ monotone and $H(e)$ disconnected for each idempotent different from 0 , and, of course, $S / M$ is not monotone.

For the final result of the paper we focus our attention on bands of groups. There were algebraically characterized by Leech [6].

DEFINITION 4.12. A compact semigroup $C$ is a band of groups if the $H$-relation is a congruence on $C$ and $C / H$ is a band.

THEOREM 4.13. A compact band of groups $C$ is monotone if and only if $\mathrm{C} / \mathrm{H}$ is monotone and the maximal subgroups of $C$ are connected.

Proof. Let $C$ be monotone. Then the necessity follows immediately from Theorems 3.2 and 4.1 . For the sufficiency, suppose that $C / H$ is monotone and the maximal subgroups of $c$ are connected. Let $a$ and $b$ 
be elements of $C / H$. Then $m_{C / H}^{-1}(a b)$ is a connected subset of $C / H \times C / H$. Defining $F: C / H \rightarrow E(C)$ by $F(e)=1_{e}$, the identity of $n^{-1}(e)$ where $n$ is the natural map from $C$ onto $C / H$, it is easily seen that $F$ is continuous. Thus $(F \times F)\left(m_{C / H}^{-1}(a b)\right)$ is a connected subset of $c \times c$. Consider the mapping $G$ from $(F \times F)\left(m_{C / H}^{-1}(a b)\right)$ into $c \times c$ defined by the following, $G\left(1_{e}, I_{g}\right)=\left(I_{e} x, y I_{g}\right)$ where $x$ and $y$ are fixed elements of $H\left(1_{a}\right)$ and $H\left(l_{b}\right)$, respectively. This mapping is also continuous and the range of $G$ will be a connected subset of $m_{C}^{-1}(x y)$ since the following relationship holds:

$$
1_{e} x y 1_{g}=1_{e}{ }^{1} g^{x y 1} e^{1} g=1_{e g} x y 1_{e g}=x y \text {. }
$$

Furthermore, the range of $G$ contains the elements $(x, y)$ and $\left(1_{e g} x, y 1_{e g}\right)$ with the latter element also contained in $\left.m_{H(1}^{-1}\right)_{e g}(x y)$. Thus there is a connected subset of $m_{C}^{-1}(x y)$ containing $(x, y)$ and $\left(I_{e g}, x y\right)$, that is, $C$ is monotone.

REMARKS. The example following Theorem 4.11 is an example of a compact connected band of groups $C$ such that $C / H$ is monotone with the maximal subgroups of $C$ nearly all disconnected. The example following Theorem 4.1 has connected maximal subgroups with $C / H$ not monotone.

\section{References}

[1] A.H. Clifford, "Unions of groups", Proceedings of the Second Florida Symposium on Automata and Semigroups, - Gainsville, Florida, 1971).

[2] A.H. Clifford and G.B. Preston, The algebraic theory of semigroups, Volume I (Mathematical Surveys, 7. American Mathematical Society, Providence, Rhode Island, 1961).

[3] T.E. Hays, "Monotone semigroups", unpublished. 
[4] J.G. Hocking and G.S. Young, Topology (Addison-Wesley, Reading, 1961).

[5] K.H. Hofmann and P.S. Mostert, Elements of compact semigroups (Charles Merrill, Columbus, Ohio, 1966).

[6] J. Leech, "The structure of band of groups", Mem. Amer. Math. Soc. 157 (1975), 67-95.

[7] M. Petrich, Introduction to semigroups (Charles Merrill, Columbus, Ohio, 1973).

Department of Mathematics,

Ohio State University,

Newark,

Ohio 43055,

USA. 